\title{
Diagnóstico da qualidade ambiental de trechos do rio Pacuí, município de Coração de Jesus, norte de Minas Gerais
}

\begin{abstract}
A água possui grande importância para todas as formas de vida existentes no planeta, por estar presente em diversos processos físicos, químicos e biológicos. Entretanto, a sociedade humana tem explorado este recurso natural de forma não sustentável, ocasionando graves problemas relacionados à baixa qualidade da água e, consequentemente, sua escassez. Diante disso, este estudo propôs realizar o diagnóstico da qualidade ambiental de um trecho do Rio Pacuí, município de Coração de Jesus, mesorregião Norte do Estado de Minas Gerais. Utilizando o índice de qualidade de água BMWP, juntamente ao Protocolo de Avaliação Rápida de Rios (PAR). A amostragem dos macroinvertebrados para aplicação do índice BMWP foram obtidas realizando arrastos de $1 \mathrm{~m}$ utilizando rede em ' $\mathrm{D}$ ', em áreas de correnteza e áreas de remanso, em um transecto de $50 \mathrm{~m}$, em 5 pontos ao longo do rio. Em cada ponto foram avaliados os 22 parâmetros estabelecidos no PAR para avaliação do ambiente e ainda foram aferidas as características físicas como largura, vazão e profundidade do rio em cada ponto. Foram identificados 1031 estabelecidos no PAR para avaliação do ambiente e ainda foram aferidas as características físicas como largura, vazão e profundidade do rio em cada ponto. Foram identificados 1031 organismos sensiveis a alterações do ambiente e Díptera que foi a ordem mais representativa em abundância. As características do rio influenciaram a abundância de macroinvertebrados, diminuindo com o aumento da vazão e aumentando com o aumento da profundidade e largura do rio. Os resultados para os índices biológicos aplicados foram correspondentes. $\mathrm{O}$ índice BMWP classificou a água dos pontos C, E e D como 'excelente', e a água dos pontos A e B como 'boa'. O PAR classificou o trecho do rio nos pontos C, E e D como 'ambiente natural', e os pontos A e B como 'ambiente alterado'. Estes resultados demonstram que os macroinvertebrados bentônicos são influenciados pelas características do rio, contudo, a qualidade do habita do entorno é o principal fator que afeta a qualidade da água na bacia do rio Pacuí. O diagnóstico da qualidade ambiental do rio Pacuí, utilizando como ferramentas índices biológicos, permite concluir que a água do trecho analisado varia entre boa e excelente, apresentando melhor qualidade nos pontos à montante da estrada principal que corta o rio na região.
\end{abstract}

Palavras-chave: Qualidade da água; Índices biológicos; Protocolo de Avaliação Rápida; Bioindicadores; Macroinvertebrados bentônicos.

\section{Diagnosis of the environmental quality of races in river Pacui, municipality of the Coração of Jesus, north of Minas Gerais}

\begin{abstract}
Water is of great importance to all life forms on the planet, being present in various physical, chemical and biological processes. However, human society has been exploiting this natural resource unsustainably, causing serious problems related to the poor quality of water resources and its scarcity. Therefore, this study proposes to diagnose the environmental quality of a river stretch called Pacuí, municipality in Coração de Jesus, North mesorregion of Minas Gerais. Using the BMWP water quality index, together with the Rapid River Assessment Protocol (PAR). Sampling of macroinvertebrates for application of the BMWP index was obtained by performing $1 \mathrm{~m}$ trawls using ' $\mathrm{D}$ ' net, in current and backwater areas, in a 50 meter transect, at five (PAR). Sampling of macroinvertebrates for application of the BMWP index was obtained by performing $1 \mathrm{~m}$ trawls using ' $\mathrm{D}$ ' net, in current and backwater areas, in a 50 meter transect, at five
points along the river. At each point the 22 parameters established in the PAR were evaluated for environmental assessment and the physical characteristics such as width, flow and depth of the river at each point were measured. It was identified 1031 invertebrates from 11 orders and 34 families. Most of the organisms found are immature insects, highlighting the orders Trichoptera, Ephemeroptera and Odonata, which are organisms sensitive to environmental changes and Diptera, which was the most representative order in abundance. River characteristics influenced the abundance of macroinvertebrates, decreasing with increasing flow and increasing with increasing river depth and width. The results for the applied biological indices were corresponding. The BMWP Index rated water from points $C, E$ and $D$ as 'excellent' and water from points $A$ and $B$ as 'good'. The PAR classified the river stretch in points $C$, E and D as 'natura environment', and points $A$ and $B$ as 'altered environment'. These results demonstrate that benthic macroinvertebrates are influenced by river characteristics. However, the quality of the surrounding hit a is the man $B$ as 'ar afecting water to conclude that the water of the analyzed stretch varies between good and excellent, presenting better quality in the points upstream of the main road that crosses the river in the region.
\end{abstract}

Keywords: Water quality; Biological index; Rapid Assessment Protocol; Bioindicators; Benthic macroinvertebrates.

\section{Topic: Engenharia Ambiental}

Reviewed anonymously in the process of blind peer.
Received: 02/02/2020

Approved: $10 / 03 / 2020$
Anielle Cristina Fonseca Pereira (iD)

Universidade Federal de Minas Gerais, Brasil

http://lattes.cnpq.br/0137614368274596

http://orcid.org/0000-0001-9561-3552

aniellecristina@gmail.com

Carlos Augusto Pereira da Silva (iD)

Instituto Federal do Norte de Minas Gerais, Brasil

http://lattes.cnpq.br/2843639478540582

http://orcid.org/0000-0001-8949-3152

carlos.augusto@ifnmg.edu.br

Flávio Pimenta de Figueiredo (DD)

Universidade Federal de Minas Gerais, Brasil

http://lattes.cnpq.br/4414228041092151

http://orcid.org/0000-0003-2839-9589

figueiredofp@yahoo.com.br
Pedro Henrique Figueiredo da Silveira (iD

Universidade Federal de Minas Gerais, Brasil

http://lattes.cnpq.br/9289460145492036

http://orcid.org/0000-0002-0665-8490

pedrohenriquebiologo@hotmail.com
Referencing this:

PEREIRA, A. C. F.; SILVA, C. A. P.; FIGUEIREDO, F. P.; SILVEIRA, P. H. F.. Diagnóstico da qualidade ambiental de trechos do rio Pacuí, município de Coração de Jesus, norte de Minas Gerais. Revista Ibero Americana de Ciências Ambientais, v.11, n.2, p.161-171, 2020. DOI: http://doi.org/10.6008/CBPC2179-6858.2020.002.0018 


\section{INTRODUÇÃO}

No decurso do processo histórico de ocupação das terras pelas sociedades, os recursos naturais sofreram grandes transformações. Práticas de uso e ocupação do solo para fins habitacionais, industriais, atividades minerarias, agricultura e pecuária tem ocupado cada vez mais território e demandado de forma desenfreada o uso demasiado dos recursos naturais, e dentre eles se destaca a água (BUZELLI et al., 2013). A água possui grande importância para todas as formas de vida existentes no planeta, por estar presente em diversos processos físicos, químicos e biológicos. Contudo, a sociedade humana tem explorado este recurso de forma não sustentável, ocasionando graves problemas relacionados à baixa qualidade dos recursos hídricos e também sua escassez (TUNDISI, 2003), principalmente decorrente do aumento da população e da ausência de políticas públicas (LUNARDI et al., 2013). Diante do exposto, a melhor gestão dos recursos naturais se faz necessária.

Os princípios da gestão ambiental constituem-se de ferramentas indispensáveis para a elaboração de planos e estratégias de manejo priorizando o equilíbrio entre a produtividade dos recursos e o desenvolvimento social. A busca por esse equilíbrio se faz por meio de técnicas de avaliação e monitoramento ambiental a fim de se garantir o controle e redução dos impactos ambientais (ESPÍNDOLA et al., 2000). Assim a gestão ambiental visa o desenvolvimento social aliado à conservação dos ambientes naturais e a recuperação de ambientes degradados (BUZELLI et al., 2013). No Brasil, tradicionalmente, o diagnóstico da saúde ambiental de um rio é realizado por meio de análises físico-químicas e microbiológicas da água. Contudo, essas metodologias retratam apenas a situação momentânea do ecossistema.

Nesse sentido, o levantamento da diversidade de hábitats aquáticos, a avaliação dos recursos tróficos disponíveis e a caracterização de macroinvertebrados bentônicos constituem-se em importantes ferramentas para a proposição de estratégias de conservação e entendimento do funcionamento dos ecossistemas aquáticos. Tais ferramentas permitem, ao avaliar a diversidade de habitats, inferir os níveis de impactos antrópicos em trechos de bacias hidrográficas. Neste contexto, os protocolos de avaliação rápida da qualidade de habitats (PAR) têm sido desenvolvidos com o objetivo de avaliar qualitativamente vários atributos dos habitats, denominados parâmetros, que são pontuados ao longo de um gradiente de ótimo à pobre, utilizando observações visuais com um mínimo de medidas. Estes protocolos são mais confiáveis porque a qualidade do habitat físico é essencial para o estabelecimento da fauna aquática, que frequentemente tem exigências específicas de habitats que são independentes da qualidade de água (CALLISTO et al., 2002; GALDEAN et al., 2000).

A habilidade de alguns organismos aquáticos, em tolerar altas concentrações de poluentes nas águas dos rios, despertou o interesse científico em sua utilização para a realização do diagnóstico da qualidade da água, denominado de índice de qualidade biológica da água (Biological Monitoring Working Party - BMWP). Este índice ordena as famílias de macroinvertebrados aquáticos em grupos, seguindo um gradiente de menor a maior tolerância dos organismos quanto à poluição orgânica (SEMA, 2019). Existem muitos indicadores biológicos para qualidade de ecossistemas aquáticos, entretanto, os mais comumente utilizados são os 
macroinvertebrados bentônicos devido à sensibilidade a poluição e às mudanças no habitat (CALLISTO et al., 2004). Os macroinvertebrados bentônicos são animais que apresentam no mínimo $0,25 \mathrm{~mm}$, habitam o sedimento de corpos d'água e têm a capacidade de colonizar diversos tipos de substrato, tais como: restos de troncos, folhas, pedras e macrófitas aquáticas, durante todo ou parte do ciclo de vida (SILVA et al., 2016).

O uso dos macroinvertebrados bentônicos, como bioindicadores ambientais, associado aos métodos tradicionais de análises, vem mostrando-se eficiente para o diagnóstico ambiental dos recursos hídricos (PIMENTA et al., 2009). Os bioindicadores são espécies cuja presença, diversidade e abundância são capazes de indicar a magnitude dos impactos ambientais em um ecossistema permitindo uma caracterização mais completa, contribuindo assim, para o manejo adequado dos recursos hídricos existentes. Existem muitos indicadores biológicos para qualidade de ecossistemas aquáticos, entretanto, os mais comumente utilizados são os macroinvertebrados bentônicos devido à sensibilidade a poluição e às mudanças no habitat (CALLISTO et al., 2004).

Em relação à tolerância frente a adversidades ambientais, podemos classificar os macroinvertebrados bentônicos em três grupos principais: organismos sensíveis ou intolerantes, organismos tolerantes e organismos resistentes. Tais características estão associadas à necessidade de oxigênio dissolvido na água, necessidade de diversidade de habitats e microhabitats, hábitos alimentares e comportamentais (GOULART et al., 2003). Vários fatores justificam o uso de bioindicadores, como o ciclo de vida relativamente longo, amostras qualitativas de fácil obtenção, metodologia desenvolvida, equipamentos simples e chaves de identificação de fácil compreensão (MONTEIRO et al., 2008). Os macroinvertebrados apresentam diversas características que os tornam excelentes bioindicadores. Por exemplo, são cosmopolitas, possuem características ecológicas bem descritas, são sedentários ou de mobilidade restrita, caracterizam a qualidade da água por certo período de tempo (a depender do ciclo de vida) e permitem avaliar as condições do sedimento e o nível do impacto através de bioacumulação e biomagnificação (MORENO et al., 2005). Tais características os colocam entre os melhores indicadores da qualidade de água em ambientes lóticos.

Como as comunidades bentônicas necessitam de certo tempo e condições próprias para estabelecer suas populações, conseguem atuar como monitores contínuos das condições ecológicas do rio, respondendo rápido às alterações. Destarte, a junção do uso de bioindicadores bentônicos e a avaliação das condições ambientais locais tornam mais eficazes o diagnóstico e o monitoramento da qualidade de um rio. Com este propósito, o objetivo deste trabalho consiste em realizar o diagnóstico da qualidade de água do Rio Pacuí utilizando índices biológicos. Concomitante a isso testaremos a hipótese de que características físicas do ambiente influenciam a qualidade da água do rio.

\section{MATERIAIS E MÉTODOS}

\section{Área de Estudo}

O estudo foi realizado na bacia do rio Pacuí, localizada na mesorregião norte do estado de Minas 
Gerais (Figura 1), entre as coordenadas $16^{\circ} 10^{\prime} 35^{\prime \prime}$ e $16^{\circ} 54^{\prime} 57^{\prime \prime}$ de latitude sul e entre $45^{\circ} 01^{\prime} 40^{\prime \prime}$ e $43^{\circ} 50^{\prime} 06^{\prime \prime}$ de longitude oeste, a $56 \mathrm{~km}$ do centro urbano de Montes Claros (Figura 2).

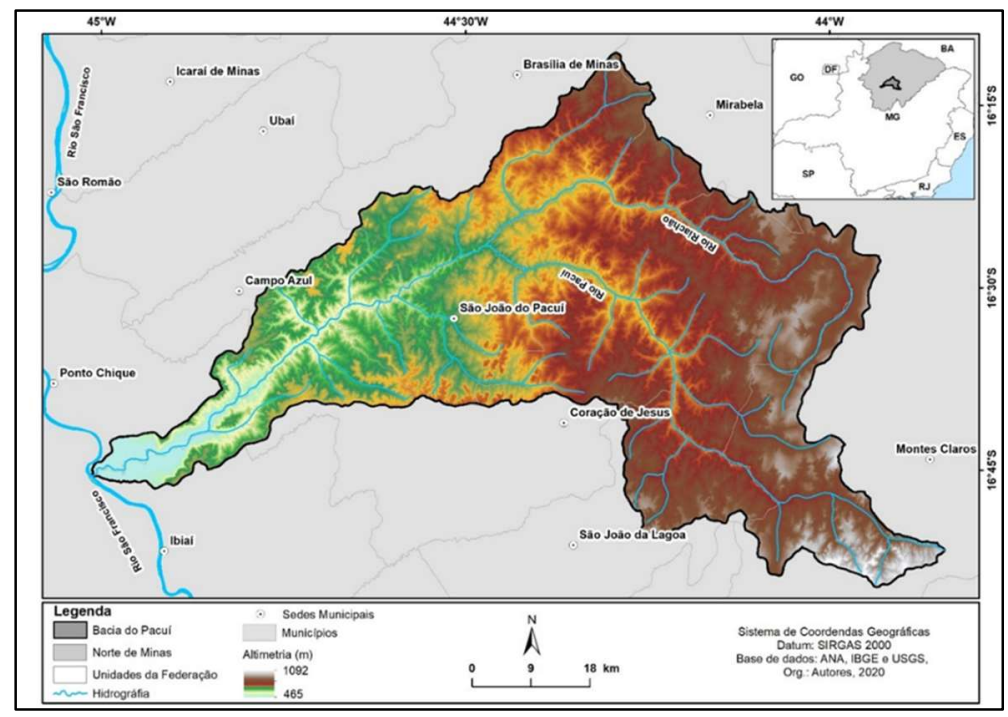

Figura 1: Mapa da bacia do rio Pacuí.

O clima da mesorregião do norte de Minas Gerais é do tipo tropical sub-úmido, próximo ao limite do sub-úmido seco, com período mais chuvoso concentrado entre os meses de novembro e janeiro e o período mais seco entre os meses de junho e agosto (NIMER et al., 1989). A vegetação predominante é o Cerrado, Campo Cerrado, Floresta Estacional Decidual Montana, Vereda e Floresta Estacional Decidual sub Montana (CARVALHO et al., 2006). A maior parte da bacia situa-se na zona rural com intenso uso da terra para a criação de bovinos. O rio principal dessa bacia possui $145 \mathrm{~km}$ desde a sua nascente, na Serra dos Fonsecas, até sua foz na margem direita do rio São Francisco, entre os municípios de Ibiaí e Ponto Chique.

\section{Métodos para diagnóstico da qualidade do rio}

A amostragem das variáveis a serem analisadas foi determinada em 5 pontos do rio Pacuí, distantes entre si em pelo menos 500 metros (Tabela 1). O ponto C (PC) está localizado abaixo da ponte por onde passa a estrada principal que corta o rio, local destinado a futura captação de água para fornecimento complementar da cidade de Montes Claros, Minas Gerais. Os pontos A e B (PA e PB) estão a jusante do PC, já os pontos E D (PE e PD) estão a montante do PC (Figura 3).

Tabela 1: Coordenadas dos pontos de coleta na bacia do rio Pacuí.

\begin{tabular}{|l|l|l|}
\hline Ponto & Latitude & Longitude \\
\hline A (Jusante) & $16^{\circ} 36^{\prime} 08.3^{\prime \prime}$ & $44^{\circ} 12^{\prime} 59.2^{\prime \prime}$ \\
\hline B (Jusante) & $16^{\circ} 36^{\prime} 22.16^{\prime \prime}$ & $44^{\circ} 12^{\prime} 53.33^{\prime \prime}$ \\
\hline C (Embaixo da ponte) & $16^{\circ} 38^{\prime} 29.3^{\prime \prime}$ & $44^{\circ} 12^{\prime} 44.5^{\prime \prime}$ \\
\hline D (Montante) & $16^{\circ} 38^{\prime} 37.5^{\prime \prime}$ & $44^{\circ} 12^{\prime} 49.03^{\prime \prime}$ \\
\hline E (Montante) & $16^{\circ} 38^{\prime} 56.2^{\prime \prime}$ & $44^{\circ} 12^{\prime} 48.4^{\prime \prime}$ \\
\hline
\end{tabular}




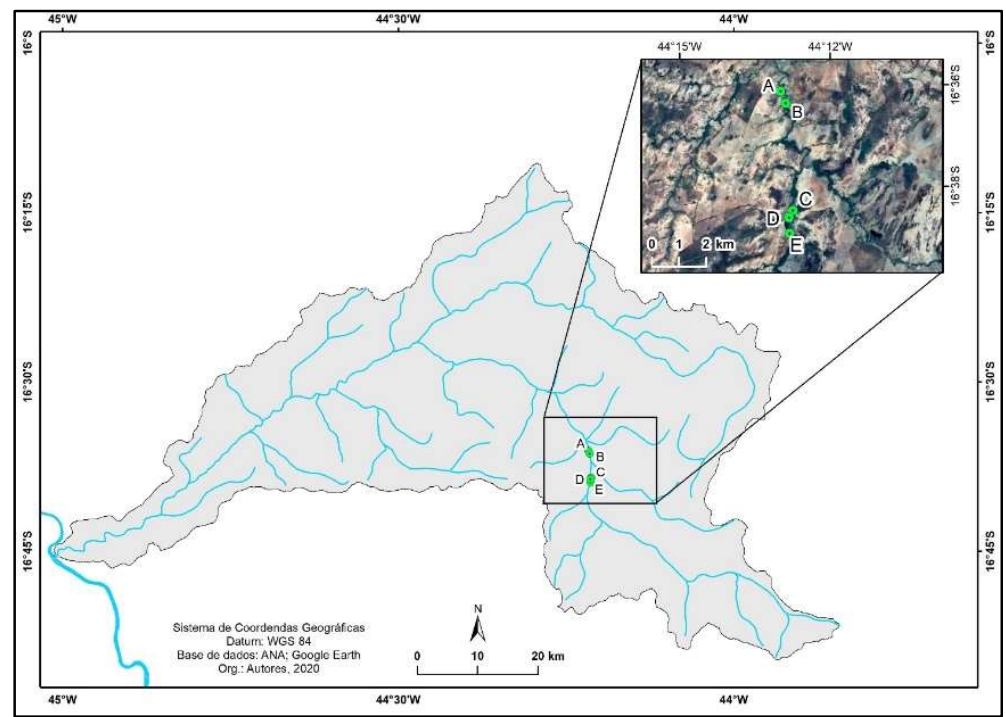

Figura 2: Imagem de satélite indicando os pontos de coleta georreferenciados.

A qualidade do hábitat foi determinada por meio da aplicação do Protocolo de Avaliação Rápida de Rios (PAR) proposto por Callisto et al. (2002). Neste protocolo são considerados 22 parâmetros que avaliam a integridade ambiental de ecossistemas de rios e riachos, levando em consideração os aspectos físicos do habitat, tais como substrato do fundo, qualidade dos remansos, características dos fluxos d'água, alteração no canal do rio, presença de mata ciliar, estabilidade das margens, entre outros. O protocolo avalia os parâmetros em categorias pontuadas de 0 a 5, atribuída a cada parâmetro com base na observação das condições do habitat. O somatório da pontuação reflete o nível de preservação das condições ecológicas do trecho da bacia estudada. Dessa forma, valores entre 0 e 40 classifica a área como 'impactada', valores entre 41 e 60 como 'alterado' e valores acima de 61 representam trecho 'natural'.

Além dos parâmetros físicos, avaliados pelo PAR, foram aferidos em cada ponto amostral a vazão $(\mathrm{L} / \mathrm{s})$, a largura $(\mathrm{m})$ e a profundidade $(\mathrm{m})$ do rio (Figura 4). Para calcular a vazão foi utilizada a técnica do flutuador, que consiste em fazer duas marcações transversais no rio, distantes entre si em $10 \mathrm{~m}$. Em seguida, soltar na primeira marcação uma garrafa pet de $500 \mathrm{ml}$, preenchida com água em $60 \%$ do seu volume, e marcar o tempo que a garrafa gasta para cruzar a segunda marcação. Em complemento, foi aferida a profundidade em 5 pontos na transversal e a largura do rio neste trecho. Após calcular a área da secção transversal, a vazão foi estimada através da Equação $1: Q=(0,8 \times L \times A) / t$, em que $Q$ é a vazão $\left(\mathrm{m}^{3} / \mathrm{s}\right), L$ é a largura do rio $(\mathrm{m}), A$ é a área da secção transversal $\left(\mathrm{m}^{2}\right)$ e $t$ é o tempo $(\mathrm{s})$.

A qualidade da água foi determinada a partir do Índice de Qualidade Biológica da Água (BMWP), adaptado por Junqueira et al. (1998). Este índice classifica as famílias de forma mais coerente, considerando as características da área de estudo abordada neste trabalho, sendo baseado na habilidade de alguns organismos aquáticos em tolerar altas concentrações de poluentes nas águas dos rios. Valores de 1 a 10 são atribuídos, em que 1 é indicativo de famílias muito tolerantes e 10 é indicativo de famílias pouco tolerantes (Tabela 2). O somatório da pontuação atribuída para cada família de macroinvertebrados classifica a água como 'excelente' para valores acima de 86, 'boa' para valores entre 64 e 85 , 'satisfatório' para valores entre 37 e 63, 'ruim' para valores entre 17 e 34 e 'muito ruim' para valores abaixo ou igual a 16. 


\section{Coleta de macroinvertebrados bentônicos}

A amostragem de macroinvertebrados bentônicos foi realizada em áreas de correnteza e remanso no rio através de arrastos de $1 \mathrm{~m}$, em um transecto de $50 \mathrm{~m}$, utilizando rede em ' $\mathrm{D}$ '. As coletas foram realizadas em 5 pontos distantes entre si em pelo menos 500 metros, como demonstrado anteriormente (Tabela 1 e Figura 3). Os sedimentos amostrados com a rede de arrasto foram armazenados em sacos plásticos identificados, contendo solução de formol a 5\% e conduzidos ao Laboratório de Ecologia e Controle Biológico de Insetos na Universidade Estadual de Montes Claros, Minas Gerais. Em laboratório, o material coletado foi lavado com água corrente em peneira granulométrica com malha de $500 \mu \mathrm{m}$, e o material retido na peneira foi triado com auxílio de lupa (ZEISS Stemi DV4, Carl Zeiss do Brasil, São Paulo). Os macroinvertebrados bentônicos encontrados foram separados e identificados em nível de família utilizando chaves de identificação.

\section{Análise dos dados}

Os dados obtidos foram analisados com auxílio de modelos lineares generalizados, seguidos de teste de resíduo, por meio do programa $R$ Development Core Team ( $R$ Foundation for Statistical Computing, Viena, Áustria, 2018), para verificar quais fatores influenciaram a qualidade da água, a comunidade bentônica e a relação entre elas e os parâmetros: vazão, largura e profundidade.

\section{RESULTADOS}

Os dados apresentados na Tabela 3 se refere aos 5 pontos analisados em relação aos parâmetros vazão, largura e profundidade. Observa-se que a maior vazão foi identificada no ponto $\mathrm{C}$ e a menor no ponto A, sendo a vazão média igual a 526,74 L/s. A largura média do rio é de 7,5 m, com maior largura determinada no trecho 1. Já a profundidade média dos pontos amostrados foi de 0,34 m, sendo o menor valor encontrado no ponto $D$.

Tabela 3: Características físicas de cada ponto amostral no rio Pacuí, Minas Gerais.

\begin{tabular}{|l|l|l|l|}
\hline Ponto & Largura $(\mathrm{m})$ & Profundidade $(\mathrm{m})$ & Vazão $(\mathrm{L} / \mathrm{s})$ \\
\hline A & 6,70 & 0,42 & 437,21 \\
\hline B & 8,00 & 0,42 & 440,75 \\
\hline C & 8,63 & 0,37 & 647,38 \\
\hline D & 7,28 & 0,19 & 509,00 \\
\hline E & 7,00 & 0,31 & 599,34 \\
\hline
\end{tabular}

A hipótese testada no estudo foi corroborada, uma vez que a classificação da qualidade do habitat apresentada pelo PAR foi correspondente ao resultado obtido para o índice BMWP, como apresentado na Tabela 4. Os métodos classificaram os pontos C, E e D como ambientes naturais e a qualidade da água como excelente, enquanto os pontos A e B foram classificados como ambientes alterados e a qualidade da água como boa. Em adição, por meio da Tabela 5 é possível verificar que foi coletado um total de 1031 macroinvertebrados, distribuídos em 11 ordens e 34 famílias. A maioria dos organismos encontrados são insetos imaturos, tendo destaque para as ordens Trichoptera, Ephemeroptera e Odonata, que são 
organismos sensíveis a alterações do ambiente. Díptera que foi a ordem mais representativa em abundância.

Tabela 4: Valores e classificação para o PAR e o BMWP para cada ponto amostrado na bacia do rio Pacuí, Minas Gerais.

\begin{tabular}{|l|l|l|l|l|}
\hline Ponto & PAR & Ambiente & BMWP & Água \\
\hline A & 57 & Alterado & 76 & Boa \\
\hline B & 59 & Alterado & 75 & Boa \\
\hline C & 63 & Natural & 107 & Excelente \\
\hline D & Natural & 99 & Excelente \\
\hline E & Natural & 89 & Excelente \\
\hline
\end{tabular}

Tabela 5: Abundância das famílias de macroinvertebrados coletados em cada ponto amostrado na bacia do rio Pacuí.

\begin{tabular}{|c|c|c|c|c|c|c|c|}
\hline Ordem & Família & A & B & C & D & $\mathrm{E}$ & Total \\
\hline Annelida & Oligochaeta & & 3 & & 5 & 5 & 13 \\
\hline Coleoptera & Belostomatidae & & & 2 & 1 & & 3 \\
\hline Coleoptera & Elateridae & & & & 1 & & 1 \\
\hline Coleoptera & Elmidae & 25 & 33 & 22 & 5 & 13 & 98 \\
\hline Coleoptera & Hydrophilidae & & & & 2 & & 2 \\
\hline Coleoptera & Naucoridae & & & 1 & & & 1 \\
\hline Coleoptera & Noteridae & & & & & 2 & 2 \\
\hline Coleoptera & Notonectidae & & & & 5 & & 5 \\
\hline Coleoptera & Psephenidae & & 37 & 6 & 3 & 1 & 47 \\
\hline Decapoda & Palaemonidae & 6 & 4 & 5 & 2 & 9 & 26 \\
\hline Díptera & Ceratopogonidae & 24 & 28 & 15 & 23 & 20 & 110 \\
\hline Díptera & Chironomidae & 109 & 137 & 53 & 36 & 61 & 396 \\
\hline Ephemeroptera & Baetidae & & 7 & & & & 7 \\
\hline Ephemeroptera & Coryphoridae & & 2 & & & & 2 \\
\hline Ephemeroptera & Euthyplociidae & 6 & 1 & & & & 7 \\
\hline Ephemeroptera & Leptophlebiidae & 5 & 4 & 16 & 10 & 3 & 38 \\
\hline Gastropoda & Caramujo & 1 & 3 & 8 & & 18 & 30 \\
\hline Hemiptera & Veliidae & 1 & & 2 & & & 3 \\
\hline Lepidoptera & Pyralidae & & & 3 & 4 & & 7 \\
\hline Odonata & Calopterygidae & 2 & 3 & 1 & & & 6 \\
\hline Odonata & Gomphidae & 4 & 7 & 10 & 2 & 2 & 25 \\
\hline Odonata & Libellulidae & & 1 & & 1 & & 2 \\
\hline Plecoptera & Perlidae & 2 & & & & & 2 \\
\hline Trichoptera & Ecnomidae & 1 & & 2 & & & 3 \\
\hline Trichoptera & Glossosomatidae & & & 6 & 3 & 1 & 10 \\
\hline Trichoptera & Helicopsychidae & & 4 & 25 & 13 & 9 & 51 \\
\hline Trichoptera & Hydrobiosidae & & & & & 1 & 1 \\
\hline Trichoptera & Hydropsychidae & 1 & 2 & 3 & 5 & 1 & 12 \\
\hline Trichoptera & Hydroptilidae & 2 & & & & & 2 \\
\hline Trichoptera & Leptoceridae & 1 & 1 & & & 1 & 3 \\
\hline Trichoptera & Odontoceridae & 14 & 22 & 19 & 12 & 4 & 71 \\
\hline Trichoptera & Philopotamidae & 1 & & 1 & 2 & & 4 \\
\hline Trombidiformes & Hydrachnidae & 1 & 2 & 5 & 2 & 4 & 14 \\
\hline Veneroida & Sphaeriidae & 15 & & 5 & 5 & 2 & 27 \\
\hline Total & & & & 210 & & 157 & 1031 \\
\hline
\end{tabular}

Os valores encontrados para riqueza de macroinvertebrados bentônicos não se diferiram estatisticamente entre os pontos $(p>0,05)$, entretanto, algumas famílias foram restritas a alguns pontos. A abundância foi maior nos trechos do rio onde a qualidade da água foi classificada como boa e menor onde a qualidade da água foi classificada como excelente (Tabela 6).

Tabela 6: Riqueza e abundância de macroinvertebrados bentônicos encontrados nos pontos amostrados do rio Pacuí, Minas Gerais.

\begin{tabular}{|l|l|l|}
\hline Ponto & Riqueza & Abundância \\
\hline A & 21 & 221 \\
\hline B & 19 & 301 \\
\hline C & 22 & 211 \\
\hline D & 21 & 142 \\
\hline E & 20 & 157 \\
\hline
\end{tabular}

As características do rio também influenciaram na abundância de macroinvertebrados. Como pode 
ser observada na Figura 5, a abundância diminuiu quanto maior a vazão (Figura 5a) e aumentou com o aumento da profundidade e da largura do rio (Figuras 5 b e $5 c$, respectivamente).

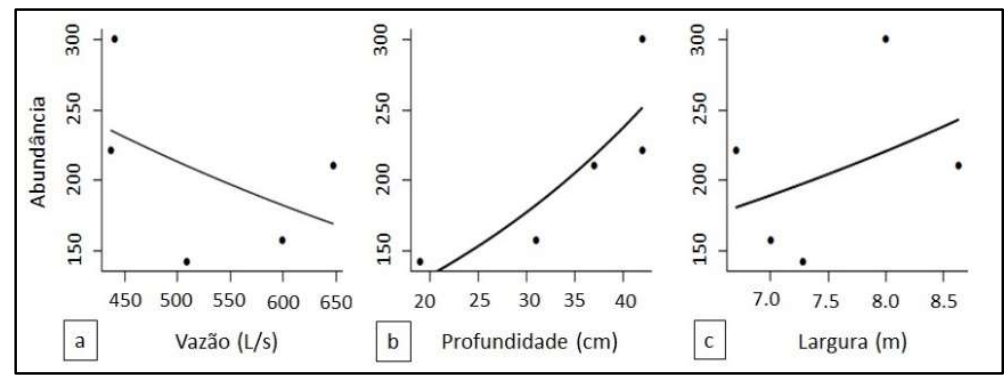

Figura 5: Relação entre a abundância de macroinvertebrados e a vazão (a), profundidade (b) e largura (c) dos pontos amostrados na bacia do rio Pacuí, Minas Gerais.

\section{DISCUSSÃO}

Os resultados apresentados na Tabela 4 comprovam a influência do habitat na qualidade da água, representada aqui pela riqueza de organismos pouco tolerante às alterações ambientais. Os pontos $A$ e $B$ estão em áreas caracterizadas por ter vários pontos de captação irregular de água, mata ciliar extremamente reduzida ou ausente, pontos de erosão, presença de pasto, cultivo de milho e tomate, e ainda leito do rio com frequentes sinais de pisoteamento do gado. Já os pontos $C$, E e D estão inseridos em áreas com um pouco menos de alterações, apesar de também apresentarem alguns pontos de erosão, a mata ciliar era mais larga e a apresentava maior distância das pastagens. Oliveira et al. (2010), em estudo realizado no município de Nova Lima, Minas Gerais, obtiveram um resultado semelhante no qual os maiores valores do índice BMWP foram registrados nos locais com presença de vegetação ripária. Da mesma forma, o trabalho desenvolvido por Monteiro et al. (2008) na bacia do rio Meia Ponte, Goiás, demonstrou que o BMWP é maior na região com menor densidade populacional, enquanto que uma menor qualidade da água foi observada onde atividades agropecuárias são intensas.

Conforme representando na Tabela 5, as famílias mais representativas foram Chironomidae (396) e Ceratopogonidae (110), isto pode estar relacionado à ocorrência cosmopolita em habitas aquáticos e semiaquáticos, além dos dípteros serem mais tolerantes a alterações ambientais, aumentando assim a sua abundância nos ambientes em relação às espécies mais sensíveis (CHANG et al., 2014). A família Naucoridae ocorreu apenas em PC; Hydroptilidae e Perlidae em PA; Baetidae e Coryphoridae em PB; Hydrobiosidae e Noteridae em PE; e Elateridae, Hydrophilidae e Notonectidae em PD. Apesar do PAR ter indicado que PA e PB são ambientes alterados e os valores do índice BMWP terem corroborado esta classificação, a presença das ordens Ephemeroptera, Plecoptera e Tricoptera, organismos sensíveis e pouco tolerantes a alterações ambientais, é um indicativo de que esses ambientes ainda conservam características naturais. Macroinvertebrados pertencentes a essas ordens são utilizados como bioindicadores de qualidade ambiental uma vez que, esses organismos precisam de elevadas concentrações de oxigênio dissolvido na água e habitam ambientes com alta diversidade de hábitats e microhábitats (GOULART et al., 2003; PIMENTA et al., 2016).

Além das famílias utilizadas na composição do BMWP, também foram amostrados um representante 
da família Trichomycteridae e um representante da ordem Cladocera em PC. A presença desses organismos também confirma o maior valor do índice em PC, classificado como ambiente natural. A família Naucoridade é composta de predadores e atua como um bioindicador de baixos níveis de enriquecimento orgânico (MAZZONI et al., 2014). Sua presença associada ao maior valor no PAR em PC demostra que a rede trófica não foi modificada. A título de exemplo, Buss et al. (2002) e Yoshida et al. (2012) encontraram menor número de indivíduos no ambiente mais preservado em estudos realizados na bacia do rio Guapimirim (Rio de Janeiro) e na cidade de Jundiaí (São Paulo), respectivamente. Catein Filho (2017) também encontrou resultado semelhante ao trabalhar na bacia do rio dos Bois, sendo a abundância de macroinvertebrados menor na ausência de mata ciliar. Tal resultado reflete a importância da matéria orgânica alóctone proveniente da vegetação ripária na estruturação desses organismos aquáticos (CUMMINS et al., 1989) e confirma a maior abundância para organismos mais tolerantes.

Apesar das características do rio Pacuí terem influenciado na abundância de macroinvertebrados, como observado na Figura 5, a diversidade de resultados disponíveis na literatura sugere que a comunidade de macroinvertebrados é mais influenciada por outros fatores como a qualidade do habitat do que pelas características do rio. Copatti et al. (2010) também encontraram maior abundância de macroinvertebrados no trecho do rio mais largo e profundo em Cruz Alta, Rio Grande do Sul. Os resultados obtidos neste estudo também corroboram em parte com o estudo de Giuliatti et al. (2009), no qual foi observada uma menor abundância de macroinvertebrados no local com maior vazão em Dourados, Mato Grosso do Sul. Entretanto, este mesmo estudo encontrou menos macroinvertebrados no local de menor profundidade. Libório (2017), por sua vez, observou uma relação positiva entre a abundância de macroinvertebrados e a largura do leito dos rios com menor declividade na região central do estado de São Paulo. Já no estudo de Callisto et al. (2001), realizado na cidade de Belo Horizonte, Minas Gerais, foi observado um aumento de até $30 \%$ na vazão do riacho estudado e, consequentemente, na largura e profundidade do mesmo. Dessa forma, o número de habitats disponíveis para os macroinvertebrados bentônicos aumentam, resultando em uma maior abundância desses organismos.

Por fim, foi constatado que a qualidade da água aumenta com a vazão do rio. Tal resultado corrobora com o estudo realizado por Silva et al. (2011) na região do Pantanal do Negro, no município de Aquidauana, Mato Grosso do Sul, onde foram encontrados valores mais elevados para o BMWP durante a cheia, quando a vazão é maior. Entretanto, Honorato et al. (2011) ao trabalhar no sul do município de Uberaba, Minas Gerais, encontraram o resultado oposto, sendo o BMWP maior no local de menor vazão. O mesmo foi observado por Peláez-Rodríguez et al. (2012) na bacia do Ribeirão Feijão, na região sudeste do estado de São Paulo, no qual a qualidade da água foi superior durante a estação seca, quando a vazão é menor. A desestabilização do rio decorrente do aumento da vazão pode aumentar o carreamento de macroinvertebrados afetando negativamente o índice BMWP (BISPO et al., 2001). Por outro lado, a menor vazão de água tende a acumular matéria orgânica favorecendo esses organismos (COPATTI et al., 2014). 


\section{CONCLUSÕES}

O diagnóstico da qualidade ambiental do rio Pacuí utilizando como ferramentas índices biológicos permitiram concluir que a água do trecho analisado varia entre boa e excelente, apresentando melhor qualidade nos pontos à montante da estrada principal que corta o rio na região. $\mathrm{O}$ presente estudo demonstrou que os macroinvertebrados bentônicos são influenciados pelas características físicas do rio, contudo, a qualidade do ambiente do entorno, como a presença de mata ciliar e a proximidade de áreas urbanas, é o principal fator que afeta a qualidade da água na bacia do rio Pacuí. Portanto, o uso do BMWP e do PAR são complementares e fornecem maior confiabilidade no diagnóstico da qualidade dos rios do que quando utilizados separadamente.

Os resultados encontrados neste trabalho podem ser utilizados como instrumento norteador para qualificar e quantificar a realidade da bacia do rio Pacuí. Assim, é possível estabelecer metas de planejamento dos recursos hídricos nos municípios banhados pelo rio, principalmente por este corpo d'água ser alvo para o fornecimento complementar do sistema de abastecimento de água da cidade de Montes Claros. Neste caso, há de se considerar que medidas mitigadoras e conservacionistas devem ser delineadas e respeitadas, uma vez foram observadas muitas interferências antrópicas no rio e no ambiente em que ele está inserido, e a captação excessiva de água pode causar uma redução drástica no volume do rio colocando em risco toda diversidade associada a ele.

\section{REFERÊNCIAS}

BISPO, P. C.; OLIVEIRA, L. G.; CRISC, V. C.; SILVA, M. M.. A pluviosidade como fator de alteração da entomofauna bentônica (Ephemeroptera, Plecoptera e Trichoptera) em córregos do Planalto Central do Brasil. Acta Limnologica Brasiliensia, Rio Claro, v.13, n.2, p.1-9, 2001.

BUSS, D. F.; BAPTISTA, D. F.; SILVEIRA, M. P.; NESSIMIAN, J. L.; DORVILLÉ, L. F. M.. Navigating wall-sized displays with the gaze: A proposal for cultural heritage. Hydrobiologia, The Hague, v.481, p.125-136, 2002.

BUZELLI, G. M.; CUNHA-SANTINO, M. B.. Análise e diagnóstico da qualidade da água e estado trófico do reservatório de Barra Bonita (SP). Ambi-Agua, Taubaté, v.8, n.1, p.186-205, 2013. DOI: http://dx.doi.org/10.4136/ambiagua.930

CALLISTO, M.; FERREIRA, W.; MORENO, P.; GOULART, M. D. C.; PETRUCIO, M.. Aplicação de um protocolo de avaliação rápida da diversidade de habitats em atividades de ensino e pesquisa (MG-RJ). Acta Limnologica Brasiliense, Rio Claro, v.14, n.1, p.91-98, 2002.

CALLISTO, M.; GONÇALVES, J. R.; MORENO, P.. Invertebrados aquáticos como bioindicadores. In: GOULART, E. M. A.. Navegando o rio das velhas das Minas aos Gerais. Belo Horizonte: UFMG, 2004. p.1-12.

CALLISTO, M.; MORETTI, M.; GOULART, M.. Macroinvertebrados bentônicos como ferramenta para avaliar a saúde de riachos. Revista Brasileira de Recursos
Hídricos, Porto Alegre, v.6, n.1, p.71-82, 2001. DOI http://dx.doi.org/10.21168/rbrh.v6n1.p71-82

CARVALHO, L. M. T.; SCOLFORO, J. R. S.; OLIVEIRA, A. D.; MELLO, J. M.; OLIVEIRA, L. T.; ACERBI-JUNIOR, F. W.; CAVALCANTI, H. C.; VARGAS FILHO, R.. Procedimentos para mapeamento. In: SCOLFORO, J. R.; CARVALHO, L. M. T.. Mapeamento e Inventário da Flora e dos Reflorestamentos de Minas Gerais. Lavras: UFLA, 2006. p.37-57.

CHANG, F. H.; LAWRENCE, J. E.; RIOS-TOUMA, B.; RESH, V. $\mathrm{H}$.. Tolerance values of benthic macroinvertebrates for stream biomonitoring: assessment of assumptions underlying scoring systems worldwide. Environmental Monitoring and Assessment, Gz Dordrecht, v.186, n.4, p.2135-2149, 2014. DOI: http://dx.doi.org/10.1007/s10661$\underline{013-3523-6}$

CATEIN FILHO, L. C.. Efeitos da perda de conectividade do componente arbóreo ripário na diversidade de insetos aquáticos em riachos. Dissertação (Mestrado em Ecologia e Evolução) - Universidade Federal de Goiás, Goiânia, 2017.

COPATTI, C. E.; MOREIRA, T. B.; MENZEL, C. A.. Environment quality assessment of a microbasin in southern Brazil through different approaches. Ambiência, Guarapuava, v.10, n.2, p.511-526, 2014. DOI:

http://dx.doi.org/10.5935/ambiencia.2014.02.06

COPATTI, C. E.; SCHIMER, F. G.; MACHADO, J. V. V..

Diversidade de macroinvertebrados bentônicos na avaliação 
da qualidade ambiental de uma microbacia no sul.

Perspectiva, Erechim, v.34, n.125, p.79-91, 2010.

CUMMINS, K. W.; WILZBACH, M. A.; GATES, D. M.; PERRY, J. B.; TALIAFERRO, W. B.. Shredders and riparian vegetation. BioScience, Oxford, v.39, n.1, p. 24-30, 1989.

ESPÍNDOLA, E. L. G.; SILVA, J. S. V.; MARINELLI, C. E.; ABDON, M. M.. A bacia hidrográfica do rio Monjolinho: uma abordagem ecossistêmica e a visão interdisciplinar. São Carlos: Rima, 2000.

GALDEAN, N.; CALLISTO, N.; BARBOSA, F. A. R.. Lotic ecosystems of Serra do Cipó, southeast Brazil: water quality and a tentative classification based on the benthic macroinvertebrates community. Aquatic Ecosystem Health \& Management, Philadelphia, v.3, n.4, p.545-552, 2000. DOI: https://doi.org/10.1080/14634980008650691

GIULIATTI, T. L.; CARVALHO, E. M.. Distribuição das assembleias de macroinvertebrados bentônicos em dois trechos do córrego Laranja Doce, Dourado/MS. Interbio, Dourados, v.3, n.1, p.4-14, 2009.

GOULART, M. D.; CALLISTO, M.. Bioindicadores de qualidade de água como ferramenta em estudos de impacto ambiental. Revista da FAPAM, Pará de Minas, v.2, n.1, p.153-164, 2003.

HONORATO, G. B. S.; PELLI, A.. Avaliação da qualidade da água em dois trechos do córrego gameleira, Uberaba-MG, com base em variáveis físico-químicas e a comunidade bentônica. SaBios-Revista de Saúde e Biologia, Campo Mourão, v.6, n.6, p.15-26, 2011.

JUNQUEIRA, V. M.; CAMPOS, S. C. M.. Adaptation of the 'BMWP' method for water quality evaluation to Rio das Velhas watershed (Minas Gerais, Brazil). Acta Limnologica Brasiliensia, Rio Claro, v.10, n.2, p.125-135, 1998.

LEITE, M. R.; FERREIRA, E. J.; LEITE, M. E.; NOBRE, B. A.; ROCHA, A. M.. Desmatamento nos municípios da bacia do rio Pacuí/MG. Revista Desenvolvimento Social, Montes Claros, v.12, n.2, p.29-40, 2014.

LIBÓRIO, R. A.. Efeitos de florestas ripárias em restauração em ecossistemas de riachos tropicais numa paisagem agrícola. Tese (Doutorado em Ciências Ambientais) Universidade Federal de São Carlos, São Carlos, 2017.

LUNARDI, J.; RABAIOLLI, J. A.. Valorização e preservação dos recursos hidrícos na busca pelo desenvolvimento rural sustentável. OKARA: Geografia em Debate, Santa Maria, v.7, n.1, p.44-62, 2013.

MAZZONI, A. C.; LANZER, R.; SCHAFER, A.. Tolerance of benthic macroinvertebrates to organic enrichment in highland streams of northeastern Rio Grande do Sul, Brazil. Acta Limnologica Brasiliensia, Rio Claro, v.26, n.2, p.119128, 2014. DOI: http://dx.doi.org/10.1590/S2179975X2014000200003

MONTEIRO, T. R.; OLIVEIRA, L. G.; GODOY, B. S. Biomonitoramento da qualidade de água utilizando macroinvertebrados bentônicos: adaptação do índice biótico BMWP' à bacia do rio Meia Ponte-GO. Oecologia Brasiliensis, La Rioja, v.12, n.3, p.553-563, 2008.

MORENO, P.; CALLISTO, M.. Bioindicadores da qualidade de água ao longo da bacia do Rio das Velhas, MG. In: Bioindicadores de qualidade de água. Belo Horizonte: UFMG, 2005. p.95-116.

NIMER, E.; BRANDÃO, A. M. P. M.. Balanço hídrico e clima da região do Cerrado. Rio de Janeiro: IBGE, 1989.

OLIVEIRA, A.; CALLISTO, M.. Benthic macroinvertebrates as bioindicators of water quality in an Atlantic forest fragment. Iheringia Série Zoologia, Porto Alegre, v.100, n.4, p.291-300, 2010.

PELÁEZ-RODRÍGUEZ, M.; MATSUMURA-TUNDISI, T.; TRIVINHO-STRIXINO, S.. Water quality assessment in Ribeirão do Feijão Basin (SP-Brazil) through benthic macroinvertebrate community. Momentos de Ciência, Florencia, v.9, n.2, p.89-96, 2012.

PIMENTA, S. M.; BOAVENTURA, G. R.; PEÑA, A. P.; RIBEIRO, T. G.. Estudo da qualidade da água por meio de bioindicadores bentônicos em córregos da área rural e urbana. Revista Ambiente \& Água, Taubaté, v.11, n.1, p.198-210, 2016. DOI: http://dx.doi.org/10.4136/ambiagua. 1672

PIMENTA, S. M.; PENA, A. P.; GOMES, P. S.. Aplicação de métodos físicos, químicos e biológicos na avaliação da qualidade das águas em áreas de aproveitamento hidroelétrico da bacia do Rio São Tomás, Município de Rio Verde - Goiás. Sociedade \& Natureza, Uberlândia, v.21, n.3 p.393-412, 2009.

SEMA. Avaliação da Qualidade da Água Através dos Macroinvertebrados Bentônicos: Índice BMWP. Curitiba: SEMA, 2019.

SILVA, F. H.; FAVERO, S.; SABINO, J.; GARNÉS, S. J. A.. Índices bióticos para avaliação da qualidade ambiental em trechos do rio Correntoso, Pantanal do Negro, Estado do Mato Grosso do Sul, Brasil. Acta Scientiarum: Biological Sciences, Maringá, v.33, n.3, p.289-299, 2011. DOI: http://dx.doi.org/10.4025/actascibiolsci.v33i3.1478

SILVA, K. W. S.; EVERTON, N. S.; MELO, M. A. D.. Application of the biological indices biological monitoring working party and average score per taxon to assess the water quality of Ouricuri river in the Municipality of Capanema, Pará State, Brazil. Revista Pan-Amazônica de Saúde, Ananindeua, v.7, n.3, p.13-22, 2016. DOI: http://dx.doi.org/10.5123/S217662232016000300002

TUNDISI, J. G.. Água no século XXI: enfrentando a escassez. São Carlos: Rima, 2003.

YOSHIDA, C. E.; ROLLA, A. P. P. R.. Ecological attributes of the benthic community and indices of water quality in urban, rural and preserved environments. Acta Limnologica Brasiliensia, Rio Claro, v.24, n.3, p.235-243, 2012. DOI: http://dx.doi.org/10.1590/S2179-975X2012005000041

A CBPC - Companhia Brasileira de Produção Científica (CNPJ: 11.221.422/0001-03) detém os direitos materiais desta publicação. Os direitos referem-se à publicação do trabalho em qualquer parte do mundo, incluindo os direitos às renovações, expansões e disseminações da contribuição, bem como outros direitos subsidiários. Todos os trabalhos publicados eletronicamente poderão posteriormente ser publicados em coletâneas impressas sob coordenação da Sustenere Publishing, da Companhia Brasileira de Produção Cientifica e seus parceiros autorizados. Os (as) autores (as) preservam os direitos autorais, mas não têm permissão para a publicação da contribuição em outro meio, impresso ou digital, em português ou em tradução. 